\title{
The effect of COVID-19 epidemic on vital signs in hospitalized patients: a pre-post heat-map study from a large teaching hospital
}

\author{
Pier Francesco Caruso ${ }^{1,2} \cdot$ Giovanni Angelotti $^{1} \cdot$ Massimiliano Greco ${ }^{1,2}\left(\mathbb{D} \cdot\right.$ Marco Albini $^{1} \cdot$ Victor Savevski $^{1}$. \\ Elena Azzolini ${ }^{1,2}$. Martina Briani ${ }^{1} \cdot$ Michele Ciccarelli ${ }^{1}$. Alessio Aghemo ${ }^{1,2} \cdot$ Hayato Kurihara $^{1}$. Antonio Voza ${ }^{1}$. \\ Salvatore Badalamenti ${ }^{1} \cdot$ Michele Lagioia $^{1}$ - Maurizio Cecconi ${ }^{1,2}$. for the Humanitas COVID-19 Task Force
}

Received: 15 September 2020 / Accepted: 27 April 2021 / Published online: 10 May 2021

(c) The Author(s), under exclusive licence to Springer Nature B.V. 2021

\begin{abstract}
The Lombardy SARS-CoV-2 outbreak in February 2020 represented the beginning of COVID-19 epidemic in Italy. Hospitals were flooded by thousands of patients with bilateral pneumonia and severe respiratory, and vital sign derangements compared to the standard hospital population. We propose a new visual analysis technique using heat maps to describe the impact of COVID-19 epidemic on vital sign anomalies in hospitalized patients. We conducted an electronic health record study, including all confirmed COVID-19 patients hospitalized from February 21st, 2020 to April 21 st, 2020 as cases, and all non-COVID-19 patients hospitalized in the same wards from January 1st, 2018 to December 31st, 2018. All data on temperature, peripheral oxygen saturation, respiratory rate, arterial blood pressure, and heart rate were retrieved. Derangement of vital signs was defined according to predefined thresholds. 470 COVID-19 patients and 9241 controls were included. Cases were older than controls, with a median age of 79 vs 76 years in non survivors $(p=<0.002)$. Gender was not associated with mortality. Overall mortality in COVID-19 hospitalized patients was $18 \%$, ranging from $1.4 \%$ in patients below 65 years to about $30 \%$ in patients over 65 years. Heat maps analysis demonstrated that COVID-19 patients had an increased frequency in episodes of compromised respiratory rate, acute desaturation, and fever. COVID-19 epidemic profoundly affected the incidence of severe derangements in vital signs in a large academic hospital. We validated heat maps as a method to analyze the clinical stability of hospitalized patients. This method may help to improve resource allocation according to patient characteristics.
\end{abstract}

Keywords COVID-19 $\cdot$ Atypical pneumonia $\cdot$ Data science $\cdot$ Data visualization $\cdot$ Heat maps $\cdot$ Vital signs

\section{Introduction}

In December 2019, a cluster of cases of atypical pneumonia caused by Severe Acute Respiratory Syndrome CoronaVirus 2 (SARS-CoV-2) was first discovered in Wuhan, capital of the Hubei region in China [1].

The outbreak represented the beginning of COVID-19 epidemic, which was subsequently declared pandemic by the World Health Organization (WHO) on March 11th, 2020 [2].

Massimiliano Greco

massimiliano.greco@hunimed.eu

1 IRCCS Humanitas Research Hospital, Via Manzoni 56, Rozzano, 20089 Milan, Italy

2 Department of Biomedical Sciences, Humanitas University, Via Rita Levi Montalcini 4, Pieve Emanuele, 20072 Milan, Italy
COVID-19 is characterized by bilateral pneumonia progressing in several cases to Acute Respiratory Syndrome (ARDS), with subsequent need for ICU admission and with a high mortality rate [1]. Survival in patients undergoing invasive mechanical ventilation has been reported to be lower than 50\% [3, 4]. Nowadays, about nine million patients were reported as infected worldwide, with a death toll of 470,000 cases [5].

In Italy, the epidemy struck since February 21th [6], expanding exponentially in the first weeks to a large number of patients requiring hospitalization, totaling more than 230,000 infected patients and 30,000 deaths today [7]. Lombardy, one of the most densely populated areas of Italy with 422 inhabitants per square kilometer, was the most affected region $[6,8]$.

To cope with the tide of incoming pneumonia cases, elective medical and surgical activity was reduced or suspended 
in the most health centers in the region, and hospitals converted part of their general wards to COVID-19 wards.

COVID-19 population admitted during this early phase of the epidemic has been described as profoundly different from normal hospital population, requiring a higher level of care $[3,9]$.

Hospitalized COVID-19 patients admitted in Lombardy presented with bilateral pneumonia with fever and cough, shortness of breath, and fatigue [10-12]. Clinical findings were matched with radiological findings of interstitial pneumonia, characterized by bilateral patchy shadows or ground glass opacity [11, 12]. Clinical findings from these patients were different from the standard hospital population, with fever as one of the most frequent finding at presentation, alteration in respiratory pattern and severe hypoxia, and a mortality rate higher than $25 \%$ in older patients [3].

Analysis of vital parameters from electronic health record have been proposed as a reliable tool to assess patient risk of deterioration, with the development of Early Warning Scores to anticipate patient deterioration, and to tailor the level of care and clinical surveillance to patient need [13, 14].

Heat maps are an informative tool to visually describe indicators of risks, and has been largely used outside the healthcare system to represent, synthesize information, and simplify decision making [15]. Recently, they have been introduced in the medical field to represent spatial distribution of events, such as cardiac arrests in a region or risk of adverse events in hospital wards [16, 17]. In Humanitas Research Center, we were undergoing research on EHR data to employ heatmaps as a synthetic tool to analyze resource allocation and needs in different wards. With the advent of COVID-19, we hypothesized that COVID-19 infection had profound impact on hospital level of care and on the incidence of severe vital sign derangements in hospitalized patient, and that this difference could be detected by risk map analysis.

\section{Methods}

We performed an Electronic Health Record (EHR) analysis study using data from Humanitas Research Hospital, a large teaching hospital in Milan, Italy. Study obtained IRB approval n. 303/20 on March 31st, 2020 and was waived from informed consent, as all patient data were anonymized before inclusion in the study.

Humanitas Research Hospital converted seven wards to COVID-19 wards to respond to Lombardy COVID-19 epidemic. All patients with laboratory confirmed COVID-19 infection admitted to seven Humanitas Research Hospital general COVID-19 wards since February 21st, 2020 to April 21st, 2020 were included. Real Time PCR on oropharyngeal swab or on lower respiratory tract aspirate was used to define laboratory confirmation of COVID-19 infection. Number of beds dedicated to COVID-19 patients increased very steadily, starting from 32 beds in the first pandemic days up to about 230 beds, distributed in seven wards, at the end of the study period. The control cohort was composed by patients admitted to the same general wards from January 1st, 2018 to December 31st, 2018 (non-COVID-19 controls). This population was a mixed surgical and medical population, including patients from pulmonology, internal medicine, nephrology, oncology, and general and abdominal surgery.

Baseline demographic data, admission data, and intrahospital transfer data were retrieved. The following vital signs were retrieved from data repository and analyzed: heart rate, blood pressure, oxygen saturation, respiratory rate, and temperature data. Vital signs are manually recorded by nurses in the electronic health record, and thus reflect patient clinical conditions confirmed by health care specialist and are not subject to false readings or transient and easily reversible clinical conditions (oxygen removal or false ECG -BP readings).

Assessment of these vitals at least every $8 \mathrm{~h}$ is the standard of care for general wards, and these data were routinely collected at predefined intervals for every patient admitted to Humanitas before and after COVID-19 pandemic. Severe derangement of vital signs was defined according to predefined thresholds: temperature above $37.5^{\circ} \mathrm{C}$, peripheral oxygen saturation below $90 \%$, respiratory rate below 10 or above 25 breaths per minute, heart rate below $40 \mathrm{bpm}$ or above $150 \mathrm{bpm}$, and mean arterial pressure below $65 \mathrm{mmHg}$.

\subsection{Statistical analysis}

Data were extracted from hospital data-warehouse and anonymized, using a predefined procedure already in place for similar datasets, and subsequently cleaned and preprocessed for analysis [18]. Continuous variables are presented as median and interquartile range (IQR) while categorical variables are expressed as frequencies and percentage with 95\% Confidence Intervals (CIs).

Wilcoxon signed-rank test and $\chi^{2}$ were used to compare continuous and categorical variables, respectively, with p-values $<0.05$ were considered statistically significant.

Heatmaps were drawn to visually express the frequency of episodes of severely deranged vital parameters in each hospital ward before and after COVID-19 pandemic. Each rectangle represents a ward and its dimension represent the cumulative number of patients admitted to the ward during the analyzed time window. Colors visually describe the percentage of vital signs which were severely outside normal range during the same time window, starting from dark green for the lowest percentages, passing by yellow up to red for the highest rates. Ward names were replaced by random numbers as ward identifiers. These identifiers, with median 
patient age, and percentage of severely deranged vital signs episodes are reported in the heatmaps for each ward.

Python environment (Python Software Foundation. Python Language Reference, version 3.8, available at http:// www.python.org) was used for statistical analysis and data visualization.

\section{Results}

A total of 470 patients were admitted to general wards in the COVID-19 phase, with 308 (65.5\%) males and $162(34.5 \%)$ females. The control population was composed by 9241 patients hospitalized in the same wards during 2018, with $4739(51.3 \%)$ males and 4502 (48.7\%) females. Median age was significantly higher in COVID-19 patients compared to control population (67 [IQR 56-77] vs 64 years [IQR 51-75], $\mathrm{p}<0.01)$. Both men and women populations were older in COVID-19 group compared to controls (67 [55-76] vs $65[52-75] \mathrm{p}=0.006$ and 68 [58-77] vs 64 [50-75], $\mathrm{p}<0.001)$ in male and females, respectively).

Mean age was 79 (73-85) in non-survivors from COVID19 population compared to 76 (66-82) in control population, $\mathrm{p}=0.002$. Median age in survivors was similar in the two groups (63 [52-72] in COVID-19 population vs 64 [IQR 51-75] in controls, $\mathrm{p}=0.7$ ).

Baseline characteristics are reported in Table 1.

In patient admitted due to COVID-19 infection, mortality was significantly associated with age (Fig. 1), p <0.001), while there was no association between mortality and gender (53/308 cases, $17.2 \%$, in men vs $32 / 163,19.6 \%$, in women, $\mathrm{p}=0.49)$.

Vital signs distribution between COVID-19 and controls and between survivors and non survivors in the two populations is reported in Table 1. Mean rate of episode of severe
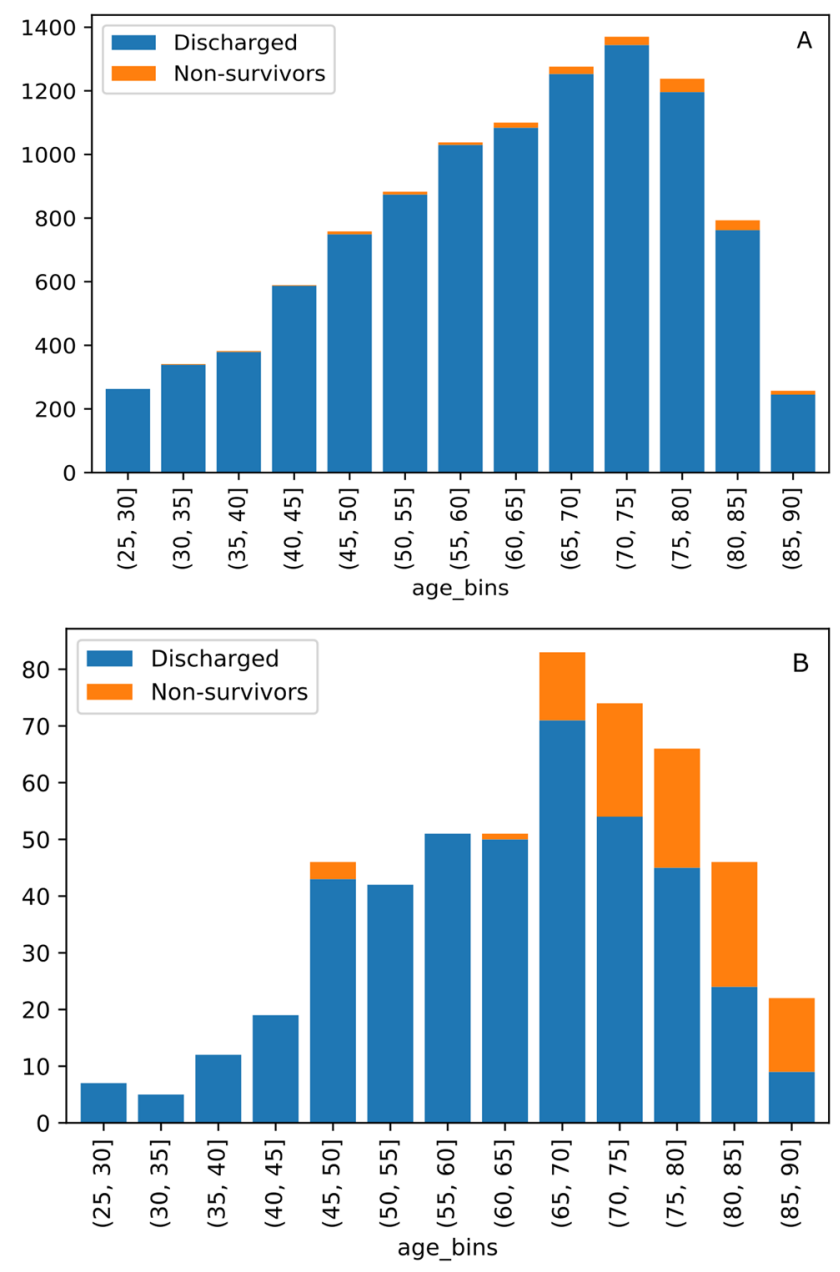

Fig. 1 Age distribution and mortality in non-COVID (a) vs COVID (b) hospitalized population

derangements in vital signs are reported in Table 2 with corresponding thresholds.

Table 1 Baseline characteristics and vital signs in COVID-19 population and controls

\begin{tabular}{|c|c|c|c|c|c|c|c|c|c|}
\hline & \multicolumn{3}{|l|}{ Survivors } & \multicolumn{3}{|c|}{ Non survivors } & \multicolumn{3}{|l|}{ Total } \\
\hline & COVID-19 & Control & $\mathrm{p}$ & COVID-19 & Control & $\mathrm{p}$ & COVID-19 & Control & $\mathrm{p}$ \\
\hline Total & 385 & 9071 & - & 85 & 170 & - & 470 & 9241 & - \\
\hline Males & $255(66 \%)$ & $4643(51 \%)$ & $<0.001$ & $53(62 \%)$ & $96(56 \%)$ & 0.35 & $308(66 \%)$ & $4739(51 \%)$ & $<0.001$ \\
\hline Females & $130(34 \%)$ & $4428(49 \%)$ & & $32(38 \%)$ & $74(44 \%)$ & & $162(34 \%)$ & $4502(49 \%)$ & \\
\hline Age & $63[52-72]$ & $64[51-75]$ & 0.705 & 79 [73-85] & $76[66-82]$ & $<0.002$ & 67 [56-77] & $64[51-75]$ & $<0.01$ \\
\hline $\mathrm{SpO}_{2}$ & $96[95-98]$ & 97[96-99] & $<0.001$ & 94 [91-97] & 96 [94-98] & $<0.001$ & 96 [94-98] & $97[96-99]$ & $<0.001$ \\
\hline Respiratory rate & $18[18,19]$ & $18[17,18]$ & $<0.001$ & $20[18-22]$ & $18[18,19]$ & $<0.001$ & $18[18,19]$ & $18[17,18]$ & $<0.001$ \\
\hline Heart rate & 78 [70-87] & 76 [68-87] & $<0.05$ & 88 [76-98] & 84 [71-98] & $<0.002$ & 79 [70-88] & 77 [68-87] & $<0.001$ \\
\hline Mean arterial pressure & 87 [80-95] & 86 [77-95] & $<0.001$ & 86 [76-96] & 80 [71-90] & $<0.001$ & 86 [80-95] & 86 [77-95] & $<0.001$ \\
\hline Temperature & 36 [36-37] & 36 [36-37] & $<0.001$ & 37 [36-37] & 36 [36-37] & $<0.001$ & 36 [36-37] & $36[36-37]$ & $<0.001$ \\
\hline
\end{tabular}

Frequencies (percentages) and median (IQR) 
The incidence of episodes of decreased oxygen saturation was significantly increased in all wards, with the highest incidence increasing from 1.19 to $4.8 \%$, and most wards reporting about a $300 \%$ increase in incidence of low oxygen saturation (Fig. 2). The number of patients affected by an acute respiratory rate event was also increased by 10 to 20 times in COVID-19 patients in some wards (from 0.54 to
$9.0 \%$ in the ward with the highest incidence of respiratory rate alterations before and during COVID), as reported in Fig. 3. The incidence of derangements in heart rate was more evenly distributed, with wards 3 and 1 reporting a reduction in episodes of alterations in COVID-19 patients, and other wards reporting an increase in heart rate alterations up to 10 times (from 0.13 to $1.41 \%$ in ward 5), Fig. 4.

Table 2 Vital signs and corresponding threshold considered to define episodes of severe vital sign derangement

\begin{tabular}{|c|c|c|c|c|c|c|c|}
\hline Vital sign & $\begin{array}{l}\text { Threshold for severe } \\
\text { derangement }\end{array}$ & $\begin{array}{l}\mathrm{N} \text { of events } \\
\text { in COVID- } \\
19\end{array}$ & $\begin{array}{l}\text { Overall rate in } \\
\text { COVID-19 popula- } \\
\text { tion* }\end{array}$ & $\begin{array}{l}\mathrm{N} \text { of events } \\
\text { in controls }\end{array}$ & $\begin{array}{l}\text { Overall rate in } \\
\text { control popula- } \\
\text { tion* }\end{array}$ & Rate ratio & $\mathrm{p}$ \\
\hline $\begin{array}{l}\text { Peripheral Oxygen Satura- } \\
\text { tion }\end{array}$ & Less than $90 \%$ & 1050 & 207.3 & 1502 & 26.3 & 7.9 & 0.001 \\
\hline Respiratory rate & $\begin{array}{l}\text { Lower than } 10 \text { per minute } \\
\text { or higher than } 25 \text { per } \\
\text { minute }\end{array}$ & 503 & 100.2 & 583 & 10.6 & 9.5 & 0.001 \\
\hline Heart rate & $\begin{array}{l}\text { Lower than } 40 \text { beats per } \\
\text { minute } \\
\text { Higher than } 140 \text { beats per } \\
\text { minute }\end{array}$ & 116 & 22.9 & 243 & 4.2 & 5.5 & 0.001 \\
\hline Mean Arterial Pressure & Lower than $65 \mathrm{mmHg}$ & 482 & 96.6 & 6979 & 121.8 & 0.8 & 0.001 \\
\hline Temperature & Higher than $37.5^{\circ} \mathrm{C}$ & 3919 & 771.9 & 13,268 & 231.0 & 3.3 & 0.001 \\
\hline
\end{tabular}

*Episode of severe vital sign instability per 1000 patient-days

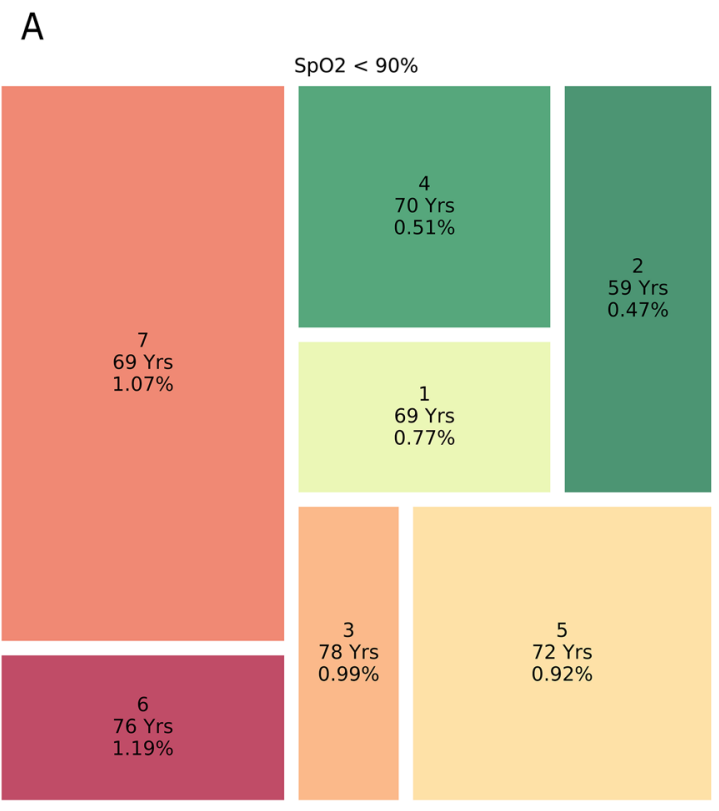

Fig. 2 Heat maps comparing peripheral oxygen desaturating events $(\mathrm{SpO} 2<90 \%)$ in pre-COVID-19 (a) vs COVID-19 hospitalized population (b). Each rectangle represents a ward, and its dimension visualizes the total number of patients admitted during the analyzed time window. The first row within every rectangle is an anonymized identifier of the ward (numbered from 1 to 7), the second line expresses the median patients' age in the department, and the last line reports
B

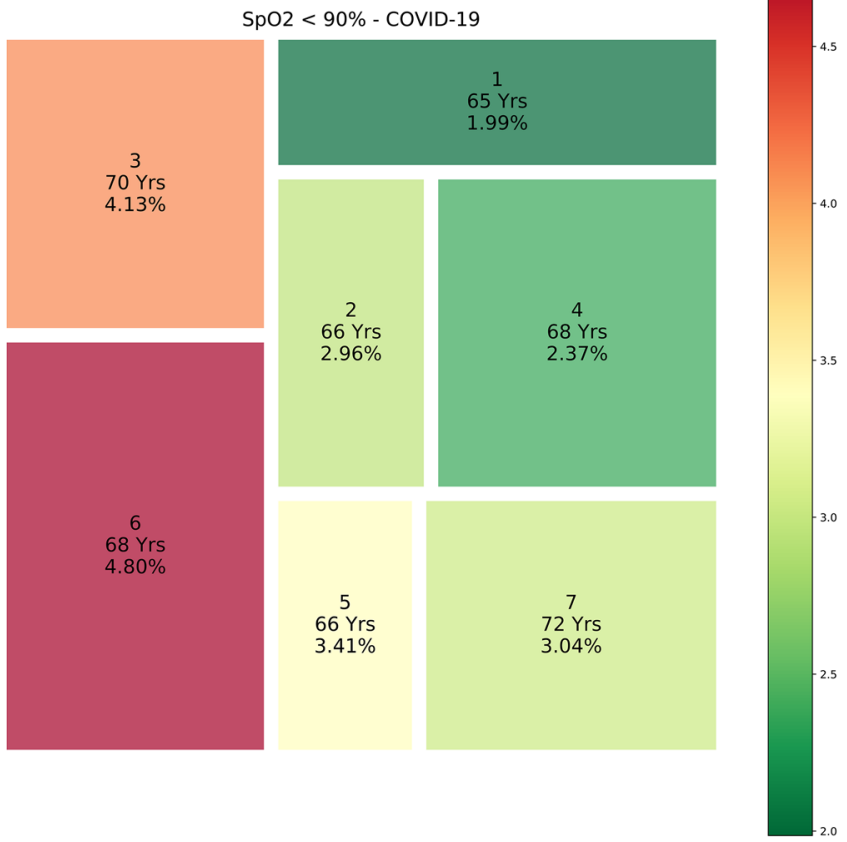

the percentage of acute oxygen desaturating events over the total number of registered measurements. Colors visually describe and compare the last line in every box with other wards. The color scale on the right links colors to the percentage of critical events over the total number of measurements. Dark green is associated to lowest percentages, contrary red to the highest percentages 


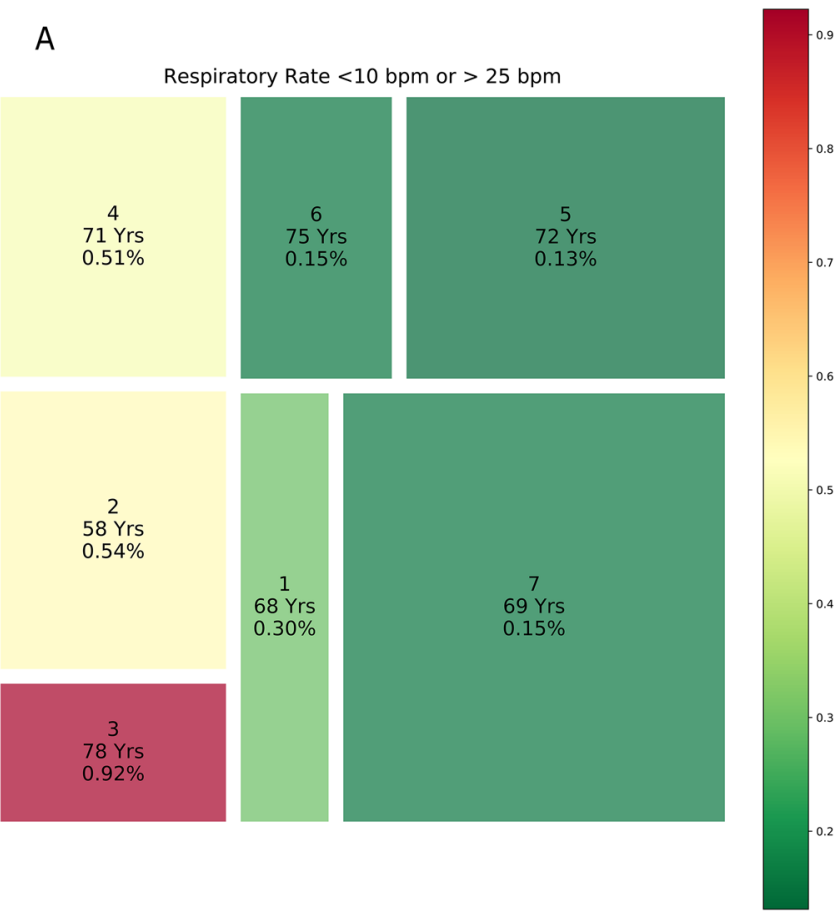

Fig. 3 Heat maps comparing severe derangements in respiratory rate (below $10 \mathrm{bpm}$ or above $25 \mathrm{bpm}$ ) in pre-COVID-19 (a) vs COVID19 hospitalized population (b). Each rectangle represents a ward, and its dimension visualizes the total number of patients admitted during the analyzed time window. The first row within every rectangle is an anonymized identifier of the ward (numbered from 1 to 7), the second line expresses the median patients' age in the department, and

Conversely, the incidence of episode of hypotension was reduced in COVID-19 patients in most wards, up to a 6 -folds reduction in ward 6 . Only ward 2 reported a slight increase in episodes of hypotension, from 1.7 to $2.3 \%$, Fig. 5. Incidence of fever was increased in patients on every ward in COVID-19 epidemic, and was the most common symptom, with about $15 \%$ incidence in the majority of wards compared to an incidence of fever below $5 \%$ in the control population (Fig. 6).

Hospital mortality in COVID-19 population was $87 / 470$ (18.1\%), with $3 / 215$ patients (1.4\%) mortality below 65 years and $82 / 256(32.0 \%), \mathrm{p}<0.001$.

\section{Discussion}

This is the first study to visually represent the effect of a COVID-19 epidemic on patient severity of illness, as measured by severe derangements in vital parameters, in a large teaching hospital. This study confirms the hypothesis that the admission of a population of patients with COVID-19 pneumonia increased patient severity and level of care in

\section{B}

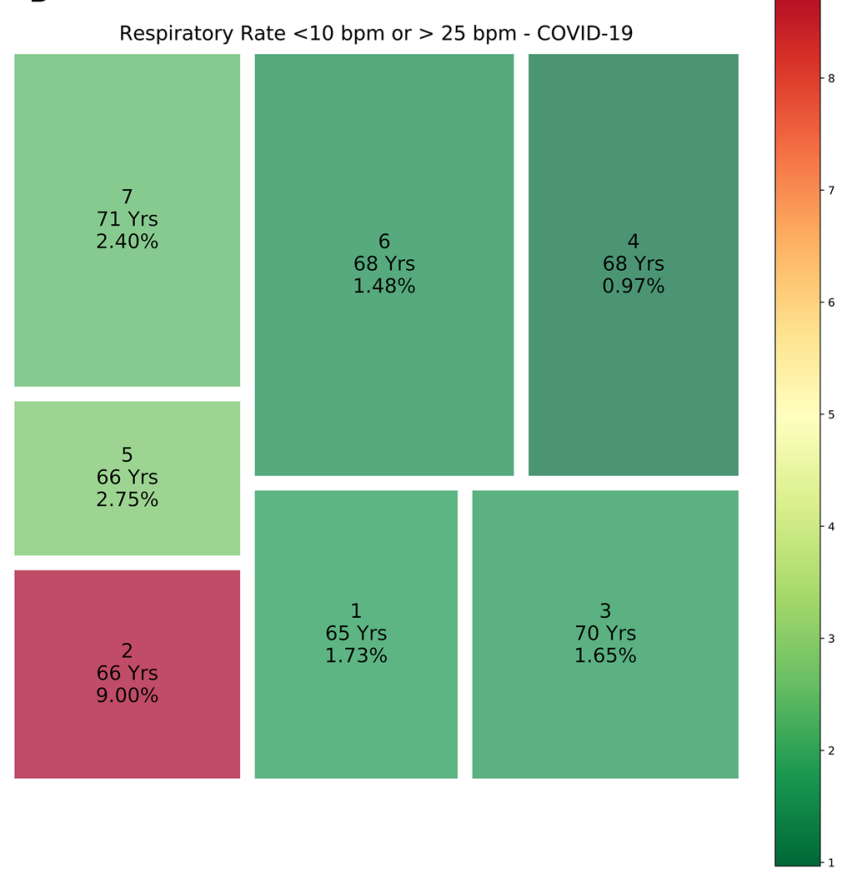

the last line reports the percentage of critical respiratory events over the total number of registered measurements. Colors visually describe and compare the last line in every box with other wards. The color scale on the right links colors to the percentage of critical events over the total number of measurements. Dark green is associated to lowest percentages, contrary red to the highest percentages.

hospital wards, due to a higher prevalence of patients with compromised vital signs.

We present a new method to visually represent incidence of clinical deterioration, using spatial distribution according to different hospital area. This method has the advantage of relaying on standard clinical data from EHR, and can be used to stratify the level of care during routine activity or, without further effort, in case of emergency, matching patients and wards according to intensity of clinical surveillance.

Heatmaps refers to spatial distribution and color representation of numbers, and are extensively used in genomics and biology, bioinformatics, but also in economics and demographical analysis $[15,19]$. Some initial study are reported in the healthcare system, as risk mapping can improve the quality and safety of the process of care [17, 19]. Lopez et al. described a prospective analysis to define risk map of adverse events in a surgical unit, to identify areas with higher risk of adverse events in the perioperative maxillofacial surgery setting [20]. In a pediatric emergency department, heat maps were based on proactive strategies involving staff interview and reactive strategies as retrospective analysis of incidents, to identify areas at 
A

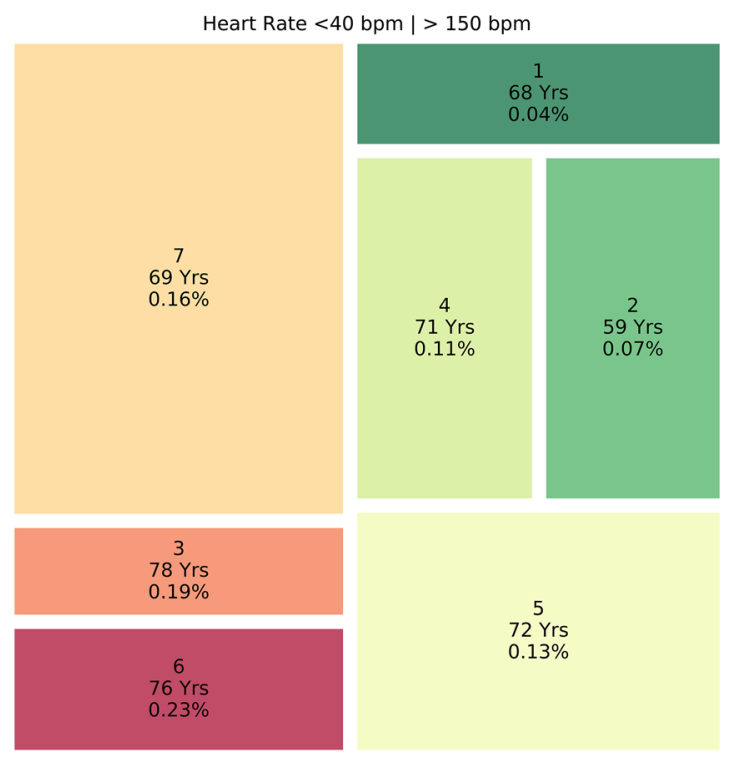

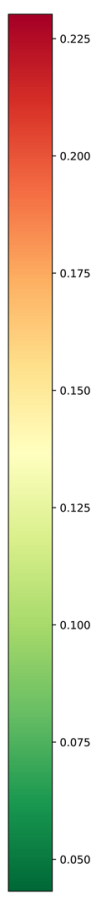

B

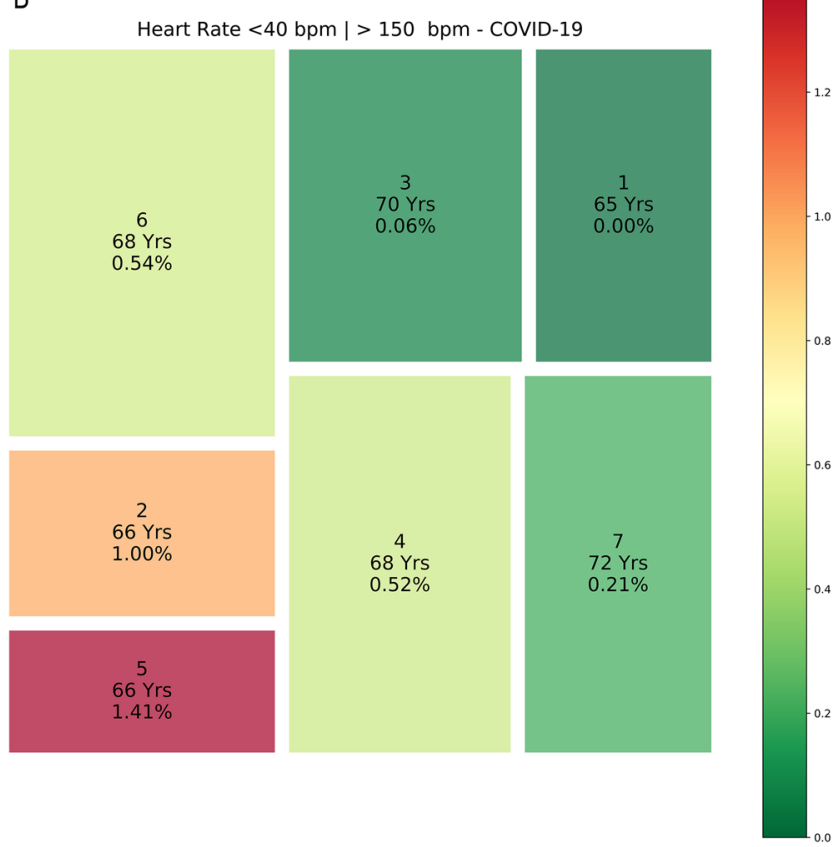

Fig. 4 Heat maps comparing severe heart rate derangements (below $40 \mathrm{bpm}$ or above $150 \mathrm{bpm}$ ) in pre-COVID-19 (a) vs COVID-19 hospitalized population (b). Each rectangle represents a ward, and its dimension visualizes the total number of patients admitted during the analyzed time window. The first row within every rectangle is an anonymized identifier of the ward (numbered from 1 to 7 ), the second line expresses the median patients' age in the department, and the

higher risk for adverse events [21]. Heat-maps have also been proposed at a regional level, to describe variation in incidence of cardiac arrest according to geographical region [16]. However, these strategies are based on a retrospective analysis of data, and are not based on the real time collection of EHR data. Spatial association may be used to simplify decision making in clinical and hospital governance, specifically in the contest of emergency and resort to surge capacity. While we did not consider exact spatial distribution and decided to anonymize the wards involved in this study, this approach can reflect exact hospital geographic distribution for internal quality improve and benchmark purposes.

The incidence of severe abnormalities in peripheral oxygen saturation and tachypnea was greatly increased in COVID-19 period. Patient hospitalized during the early phase of COVID-19 epidemic presented almost invariably with bilateral interstitial pneumonia. Oxygen saturation is associated with severity of COVID-19 disease, with up to $20 \%$ of patients presenting with peripheral oxygen saturation below 90\% [3], a finding confirmed in ARDS from other etiologies [22]. Our data demonstrate the severe derangement in oxygen saturation in hospitalized COVID-19 patients. last line reports the percentage of critical cardiac frequency derangements over the total number of registered measurements. Colors visually describe and compare the last line in every box with other wards. The color scale on the right links colors to the percentage of critical events over the total number of measurements. Dark green is associated to lowest percentages, contrary red to the highest percentages

Episodes of hypotension were reduced in COVID-19 population compared to the control population. In patients subjected to surgery and in medical patients before COVID19 era, hypotensive episodes were frequent. In the reference group, they ranged from 1.8 to $6 \%$. Conversely, shock is rare in COVID-19 patients, and has been reported as low as $1 \%$ in patients outside ICU in previous study, compared to $30 \%$ of patients admitted to ICU with severe hypoxia and need for mechanical ventilation $[1,12]$. Even if hypotension is an ominous sign associated with serious prognosis in COVID-19 infection [10, 23], its incidence in non-severe cases is rare, and a large cohort study from China found $0.1 \%$ incidence in hospitalized and outpatients COVID-19 infection [24].

Fever is one of the most common symptoms in patients with COVID-19 infection, almost invariably reported in hospitalized patients [12]. Fever over $38{ }^{\circ} \mathrm{C}$ was present in at least one third of COVID-19 patients at presentation [12]. Our data confirms fever as the most frequent vital sign in COVID-19 population, with 771 rate per 1000 patient days and a threefold increase compared to the control population.

Despite increased risk of clinical deterioration, reflected by increased frequency of derangement in parameters, the large majority of vital signs were in the range of normality 


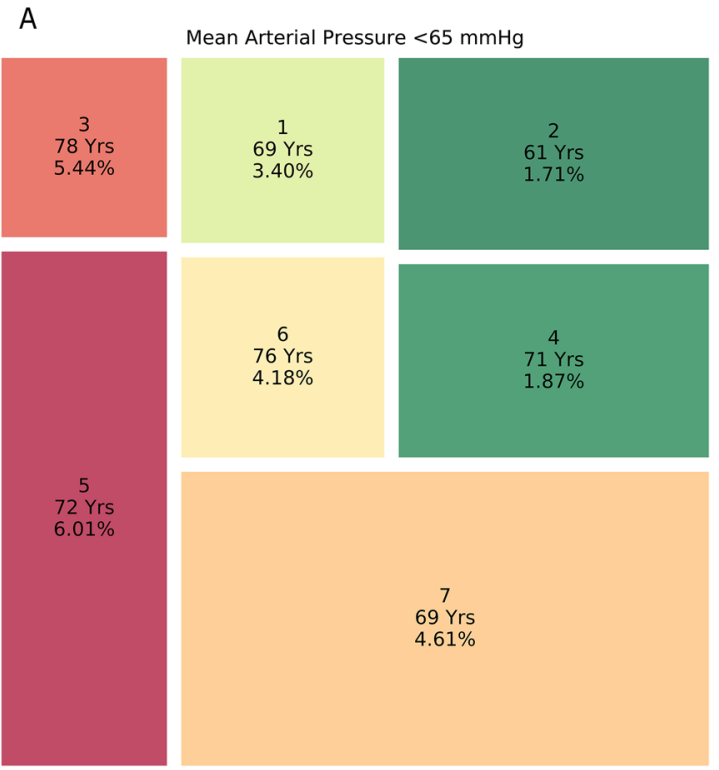

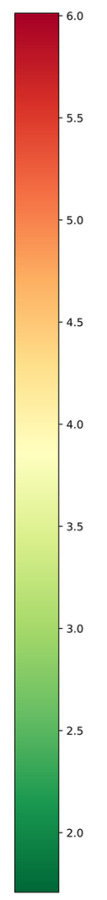

B

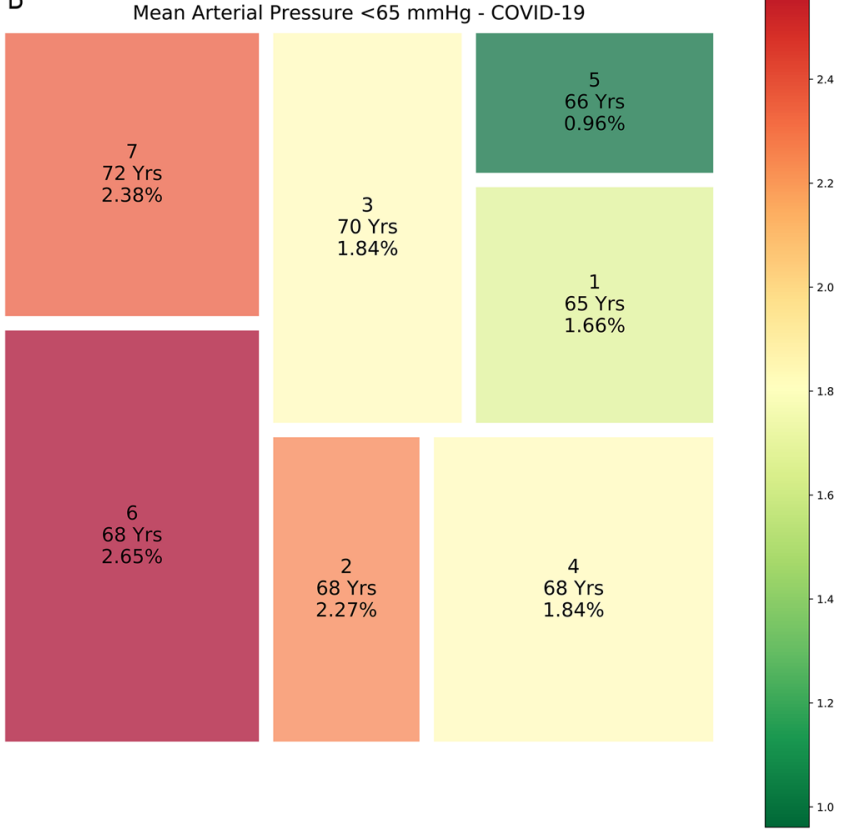

Fig. 5 Heat maps comparing hypotension (MAP $<65 \mathrm{mmHg}$ ) in pre-COVID-19 (a) vs COVID-19 hospitalized population (b). Each rectangle represents a ward, and its dimension visualizes the total number of patients admitted during the analyzed time window. The first row within every rectangle is an anonymized identifier of the ward (numbered from 1 to 7 ), the second line expresses the median patients' age in the department, and the last line reports the percent-

in both COVID-19 and non-COVID-19 patients, as reported in Table 1, revealing that with appropriate care COVID19 bilateral pneumonia was safely managed in most cases. In this context, prediction of clinical deterioration come to considerable importance.

Patient clinical deterioration is often anticipated by changes in vital signs; accordingly, clinical scores have been developed to identify patients at risk of clinical deterioration and are commonly used in clinical practice. Early warning systems is one of those score, developed on seven different parameters, and demonstrated good discriminatory performance in identification of patients at increased risk of death [25]. Similar scores have also been implemented not only to predict clinical deterioration, but to allocate patient according to the appropriate level of clinical surveillance [26]. Goldfrad demonstrated that a reduction in surveillance was associated with an increased risk of mortality even in stable patient discharged at night from ICU, compared to the control population discharged during dayshift [27]. The stratification of hospital areas according to their clinical severity and to the risk of clinical deterioration is fundamental to allocate resources and improve patients' safety, specifically in an emergency setting due to a large number of patients presenting with age of critical hypotensive events over the total number of registered measurements. Colors visually describe and compare the last line in every box with other wards. The color scale on the right links colors to the percentage of critical events over the total number of measurements. Dark green is associated to lowest percentages, contrary red to the highest percentages

pneumonia and respiratory distress. To cope with COVID19 epidemic, the hospital reorganized general wards in COVID-19 wards with different levels of care. As ICU surge capacity was strained in the whole Lombardy region, some wards were transformed in intermediate units (level 2), with frequent use of CPAP and NIV [6, 28]. Different levels of patient severity are indeed appreciable from our analysis, with wards 6 and 5 having the highest number of episodes of tachypnea (9.0\% and $2.75 \%)$ and desaturation (4.8\% and $3.4 \%$ ) in the COVID-19 phase.

COVID-19 patients were generally older than control population, in both male and female populations. Median age was similar in survivors between COVID-19 and controls (63 and 64 years respectively), while COVID-19 patients who did not survive had a median increase of three years compared to non survivors in controls (79 vs 76 years). Gender was not associated with mortality in our population.

In this study we report a $18 \%$ mortality in hospitalized patients, with a mortality $1.4 \%$ below 65 years and about $30 \%$ for patients over 65 years. This is similar to the large cohort of patients described by Richardson and colleagues, reporting $26.6 \%$ mortality in non-ventilated patients over 65 years in New York outbreak [3], and confirms the severity of COVID-19 infection in hospitalized patients. 
A

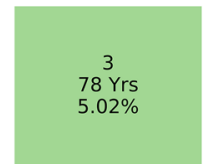

Temperature $>37.5^{\circ} \mathrm{C}$
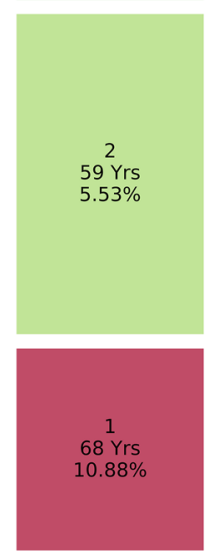

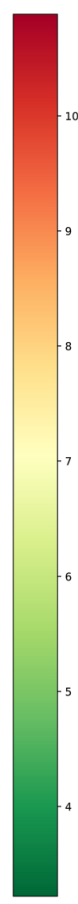

Fig. 6 Heat maps comparing temperature measurements in preCOVID-19 (a) vs COVID-19 hospitalized population (b). Any patient's temperature greater than or equal to $37.5^{\circ} \mathrm{C}$ was considered abnormal. Each rectangle represents a ward, and its dimension visualizes the total number of patients admitted during the analyzed time window. The first row within every rectangle is an anonymized identifier of the ward (numbered from 1 to 7 ), the second line expresses
B

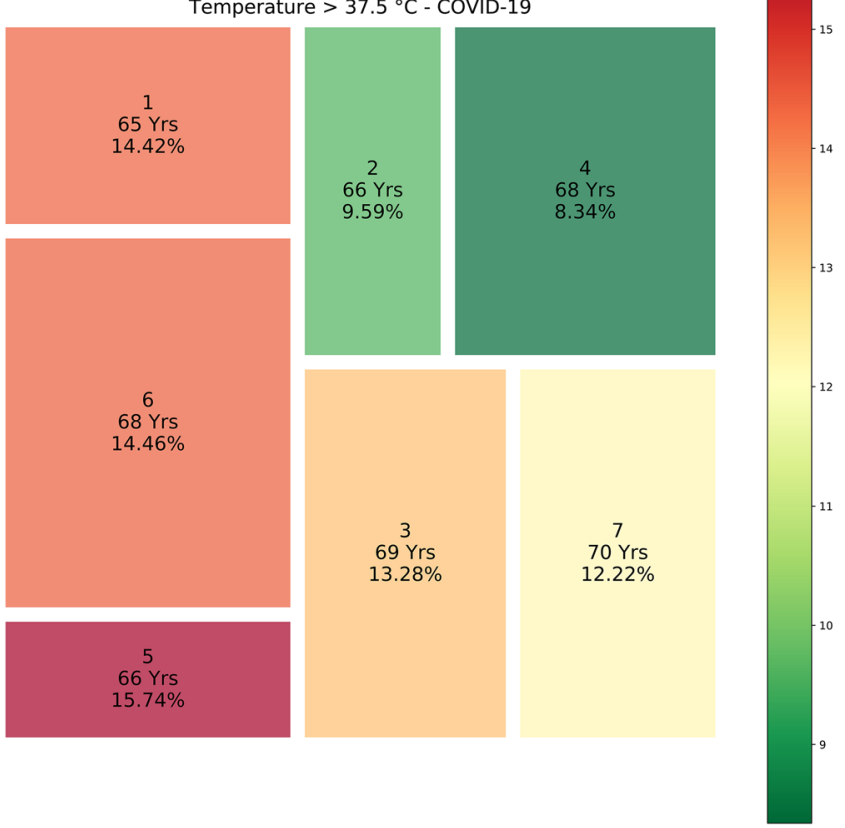

the median patients' age in the department, and the last line reports the percentage of abnormal temperature events over the total number of registered measurements. Colors visually describe and compare the last line in every box with other wards. The color scale on the right links colors to the percentage of critical events over the total number of measurements. Dark green is associated to lowest percentages, contrary red to the highest percentages

\section{Conclusion}

We used Electronic Health Record data routinely collected in general wards in our hospital, retrieving a predefined set of vital signs which represent the standard monitoring outside ICU. More detailed data, including patient baseline comorbidities, drug administration, and interventions during hospitalization, could not be retrieved. While this strategy limit research findings, this approach as the advantage of presenting a low-cost system to map patients and intensity of care in general wards. Pre-post study bear limitations in their design, due to confounding factors. In our study, we did not match patients by baseline characteristics, a technique used to improve comparison, to preserve our primary research question on the impact COVID-19 pandemic had on hospital organization. In this specific setting of an epidemic of bilateral pneumonia arising from the very same origin, we believe pre-post design respond to our research question on how COVID-19 affected the structure and function of hospitals in Lombardy, according to patient severity and increased level of care.

The large number of COVID-19 patient admitted in the early phase of Lombardy epidemic had a great impact on hospital organization and patient clinical severity, has demonstrated by risk-map analysis. Incidence of severe respiratory derangements and frequency of fever were the parameters more frequently affected. Heat maps are a simple and effective to map patient risk and ward intensity of care to improve resource allocation and increase patient safety, and quality of care in standard and emergency settings.

\section{Declarations}

Conflict of interest The authors declare that they have no conflict of interest.

Informed consent This research study was conducted retrospectively from EHR data obtained for clinical purposes. The IRB of Humanitas Research Hospital approved this study (IRB approval 303/20), 
and waived informed consent due to the retrospective evaluation of anonymized clinical data.

\section{References}

1. Huang C, et al. Clinical features of patients infected with 2019 novel coronavirus in Wuhan, China. Lancet. 2020;395:497-506.

2. WHO announces COVID-19 outbreak a pandemic. 2020. https://www.who.int/director-general/speeches/detail/whodirector-general-s-opening-remarks-at-the-media-brief ing-on-covid-19---11-march-2020.

3. Richardson S, et al. Presenting characteristics, comorbidities, and outcomes among 5700 patients hospitalized with COVID-19 in the New York City Area. JAMA. 2020;323:2052-9.

4. Yang X, et al. Clinical course and outcomes of critically ill patients with SARS-CoV-2 pneumonia in Wuhan, China: a singlecentered, retrospective, observational study. Lancet Respir Med. 2020;8:475-81.

5. John Hopkins. https://coronavirus.jhu.edu/map.html. https://www. worldometers.info/coronavirus.

6. Grasselli G, Pesenti A, Cecconi M. Critical care utilization for the COVID-19 Outbreak in Lombardy, Italy: early experience and forecast during an emergency response. JAMA-J Am Med Assoc. 2020;323:1545.

7. COVID-19 ITALIA - desktop. https://opendatadpc.maps.arcgis. com/apps/dashboards/b0c68bce2cce478eaac82fe38d4138b1.

8. ISTAT. Annuario statitistico italiano, Annu. Istat. 2019. https:// www.istat.it/it/archivio/236772.

9. Grasselli G, et al. Baseline characteristics and outcomes of 1591 patients infected with SARS-CoV-2 admitted to ICUs of the Lombardy region, Italy. JAMA. 2020;323:1574-81.

10. Zhou F, et al. Clinical course and risk factors for mortality of adult inpatients with COVID-19 in Wuhan, China: a retrospective cohort study. Lancet. 2020;395:1054-62.

11. Chen N, et al. Epidemiological and clinical characteristics of 99 cases of 2019 novel coronavirus pneumonia in Wuhan, China: a descriptive study. Lancet. 2020. https://doi.org/10.1016/S01406736(20)30211-7.

12. Wang D, et al. Clinical characteristics of 138 hospitalized patients with 2019 novel coronavirus-infected pneumonia in Wuhan, China. JAMA—J Am Med Assoc. 2020;323:1061-9.

13. Jayasundera R, Neilly M, Smith T, Myint P. Are early warning scores useful predictors for mortality and morbidity in hospitalised acutely unwell older patients? A systematic review. J Clin Med. 2018;7(10):309.
14. Goldhill DR, McNarry AF, Hadjianastassiou VG, Tekkis PP. The longer patients are in hospital before intensive care admission the higher their mortality. Intensive Care Med. 2004;30(10):1908-13.

15. Von Mettenheim HJ, Breitner MH. Decision analytics with heatmap visualization for multi-step ensemble data. Bus Inf Syst Eng. 2014;6(3):131-40.

16. Peluso S, et al. A Bayesian spatiotemporal statistical analysis of out-of-hospital cardiac arrests. Biom J. 2020;62:1105-19.

17. Samartín-Ucha M, et al. Devising of a risk map on the management of high risk alert medication in a high level university hospital. Farm Hosp. 2019;43(3):110-5.

18. Johnson AEW, et al. MIMIC-III, a freely accessible critical care database. Sci Data. 2016. https://doi.org/10.1038/sdata.2016.35.

19. Moore N, Middleton PM, Ren S. Lost capacity in emergency departments and its economic implications: a simulation study and economic analysis. Emerg Med Australas. 2020;32:974-9.

20. Sánchez López JD, Cambil Martín J, Villegas Calvo M, Toledo Páez MA, Cariati P, Moreno Martín ML. Development of a risk map in an oral and maxillofacial surgical unit. J Healthc Qual Res. 2019;34(4):209-16.

21. Mojica E, Izarzugaza E, Gonzalez M, Astobiza E, Benito J, Mintegi S. Elaboration of a risk map in a paediatric emergency department of a teaching hospital. Emerg Med J. 2016;33(10):684-9.

22. Bellani G, et al. Epidemiology, patterns of care, and mortality for patients with acute respiratory distress syndrome in intensive care units in 50 countries. JAMA. 2016;315(8):788-800.

23. Bhatraju PK, et al. Covid-19 in critically ill patients in the Seattle region-case series. N Engl J Med. 2020. https://doi.org/10.1056/ NEJMoa2004500.

24. Guan WJ, et al. Clinical characteristics of coronavirus disease 2019 in China. N Engl J Med. 2020;382:1708-20.

25. Liljehult J, Christensen T. Early warning score predicts acute mortality in stroke patients. Acta Neurol Scand. 2016;133(4):261-7.

26. Cei M, Bartolomei C, Mumoli N. In-hospital mortality and morbidity of elderly medical patients can be predicted at admission by the modified early warning score: a prospective study. Int J Clin Pract. 2009;63(4):591-5.

27. Goldfrad C, Rowan K. Consequences of discharges from intensive care at night. Lancet. 2000;355(9210):1138-42.

28. Carenzo L, et al. Hospital surge capacity in a tertiary emergency referral centre during the COVID-19 outbreak in Italy. Anaesthesia. 2020. https://doi.org/10.1111/anae.15072.

Publisher's Note Springer Nature remains neutral with regard to jurisdictional claims in published maps and institutional affiliations. 